\title{
EL PROCESO DE TRANSFORMACIÓN MILITAR EN ESPAÑA
} (2004-2011)

\author{
Guillem Colom ${ }^{1}$ \\ Universidad Pablo de Olavide \\ Instituto General Gutiérrez Mellado (UNED)
}

\begin{abstract}
Resumen:
El artículo realiza un análisis de la Transformación militar española desde sus inicios hasta la actualidad. Tras la consolidación de este proceso en Estados Unidos en el 2001 y en la Alianza Atlántica dos años después, España inició la Transformación militar en el año 2004 para adaptar la estructura de fuerzas y catálogo de capacidades militares para satisfacer los retos actuales y futuros, garantizando así la continuidad del proceso de modernización material, la profesionalización de las fuerzas armadas, la adaptación de la arquitectura defensiva del país al mundo de la posguerra fría y de la integración española en las organizaciones de seguridad colectivas.
\end{abstract}

Palabras clave: Política de Defensa, Política Militar, Transformación, España, Fuerzas Armadas.

Title in English: "The Process of Military Transformation in Spain”.

\begin{abstract}
:
The article analyses the Spanish military Transformation from its beginnings until the present day. In 2004 - one year after the launch of this process in the Atlantic Alliance and three years after its consolidation in the United States - Spain initiated its defence Transformation as a means to prepare the force structure and military capabilities of its armed forces to meet the current and future challenges, thus ensuring the continuity of the process of modernization of materiel, the professionalization of the military, the adaptation of the security architecture of the country to the post-Cold War environment and the Spanish integration to the collective security organizations.
\end{abstract}

Keywords: Defence Policy, Military Policy, Transformation, Spain, Armed Forces.

Copyright $@$ C UNISCI, 2011.

Las opiniones expresadas en estos artículos son propias de sus autores, y no reflejan necesariamente la opinión de UNISCI. The views expressed in these articles are those of the authors, and do not necessarily reflect the views of UNISCI.

\footnotetext{
${ }^{1}$ Guillem Colom Piella es Doctor en Paz y Seguridad Internacional (IUGGM-UNED), Master y doctorando en Relaciones Internacionales (UAB), Licenciado en Sociología (UAB) y en Ciencias Politicas (UAB). Profesor del área de Ciencia Política de la Universidad Pablo de Olavide y del Instituto General Gutiérrez Mellado (UNED). Líneas de investigación: seguridad y defensa, relaciones transatlánticas, asuntos militares, estudios estratégicos.Email: guillemcolom@hotmail.com, gcolpie@upo.es. 


\section{Introducción}

Hoy en día, son muchos los ministerios de defensa de todo el globo que se hallan inmersos en ambiciosos procesos de reorganización de sus estructuras, racionalización de sus funciones, flexibilización de sus procesos y mejora de sus competencias con objeto de satisfacer los requerimientos presentes y futuros. Este proceso orientado a acomodar la arquitectura de seguridad, defensa y militar a los retos del siglo XXI recibe el nombre de Transformación y se ha convertido en el eje de las políticas de defensa y militares de todas las naciones avanzadas.

La Transformación, concebida inicialmente como el proceso mediante el cual se conquistaría una Revolución en los Asuntos Militares (RMA) que garantizaría victorias rápidas, decisivas, limpias y sin apenas daños colaterales ${ }^{2}$, alcanzó fama mundial tras el nombramiento de Donald Rumsfeld como Secretario de Defensa de Estados Unidos. No obstante, fueron los trágicos acontecimientos del 11 de Septiembre de 2001 los que acabaron con la aparente "pausa estratégica" iniciada tras la caída del Telón de Acero y convencieron a muchas potencias de la urgencia de adaptar sus entramados de defensa - grandes, rígidas y burocratizadas estructuras diseñadas para combatir en un conflicto convencional o nuclear contra el Pacto de Varsovia - para satisfacer los retos del mundo del siglo XXI; por lo que este proceso pasó a vertebrar los procesos de planeamiento de la defensa del grueso de los países avanzados.

Aunque los triunfos iniciales en Afganistán e Iraq parecieron confirmar la validez de las transformaciones realizadas hasta la fecha; el paso de las operaciones convencionales a los cometidos de seguridad, estabilización y contrainsurgencia revelaron las vastas carencias de los ejércitos actuales en escenarios de baja o media intensidad y pusieron de manifiesto la necesidad de redefinir la Transformación militar, un proceso que a fecha de hoy busca dotar a unas fuerzas armadas todavía organizadas, adoctrinadas y equipadas para la lucha convencional, de las capacidades necesarias para resolver los problemas actuales (guerra irregular, híbrida, informativa y ciberguerra, estabilización, apoyo militar a la reconstrucción, ocupación o seguridad $)^{3}$ y preparar la arquitectura defensiva de los países avanzados para anticiparse a los peligros futuros.

Sin embargo, la adaptación de unas estructuras de seguridad proyectadas para enfrentarse al Pacto de Varsovia, a otras capaces de satisfacer los retos presentes y anticiparse a los peligros futuros es un proceso largo y complejo que requiere ajustar los recursos y medios dedicados a la defensa conforme a la evolución y la tendencia previsible de la amenaza. Es por ello que el proceso de Transformación de la defensa no sólo requiere adaptar la estructura de fuerzas, el catálogo de capacidades o los patrones de despliegue de las fuerzas

\footnotetext{
${ }^{2}$ Una RMA se define como un profundo cambio en la forma de combatir que, motivada por la integración de nuevas tecnologías, doctrinas, formas de organización de la fuerza y conceptos que guían su empleo en operaciones, convierte en irrelevante u obsoleto el estilo militar anterior. En la década de 1990, esta posibilidad motivada por la aplicación de las tecnologías de la información y las comunicaciones en la esfera militar articuló el análisis estratégico estadounidense e internacional. Un análisis detallado de esta idea puede hallarse en: Shimko, Keith L. (2010): The Iraq Wars and America's Military Revolution, Nueva York, Cambridge University Press o Gray, Colin S. (2002): Strategy for Chaos: Revolutions in Military Affairs and the Evidence of History, Portland, Frank Cass.

${ }^{3}$ En el ámbito aliado, una capacidad militar combina Doctrina, Organización, Adiestramiento, Material, Liderazgo y Educación, Personal, Instalaciones e Interoperabilidad (NATO Standardization Agency (2010): AAP-6 Glossary of Terms and Definitions, Bruselas, OTAN, en www.nato.int/docu/stanag /aap006/aap-62010.pdf). No obstante, en nuestro país se entiende como el conjunto de Material, Infraestructuras, Recursos Humanos, Adiestramiento, Doctrina y Organización (MIRADO).
} 
armadas; sino también realizar cambios en la concepción estratégica, las políticas de personal civil y militar, los proyectos de investigación y desarrollo, los procesos de trabajo, la gestión financiera o la cooperación industrial, entre otros elementos.

A pesar de que Estados Unidos ha sido el promotor y principal valedor de la Transformación, son muchos los países de nuestro entorno que están realizando sendos procesos, condicionados tanto por la necesidad de adaptar sus arquitecturas de seguridad y defensa al mundo actual, como por la exigencia de mantener la ventaja cualitativa frente a sus potenciales adversarios y la paridad de capacidades con relación a sus socios y aliados.

España no ha sido una excepción. Condicionada por factores externos como la rápida evolución del entorno estratégico y por su pertenencia a la Alianza Atlántica; e internos como la profesionalización del ejército y la modernización del material, nuestro país emprendió formalmente la Transformación de las fuerzas armadas en el año 2004 con el objeto de adaptar su estructura de fuerzas y catálogo de capacidades militares a los retos que plantea el mundo del siglo XXI. Además, el arranque de este proceso coincidió con el comienzo de una etapa de gran producción normativa que, necesaria para enmarcar el proceso de Transformación militar, pretende reformar la concepción, estructura y funcionamiento de la política de defensa y militar española para adaptarla a los cánones políticos y estratégicos vigentes.

En consecuencia, aunque la Transformación debería afectar al conjunto de la defensa española (la concepción estratégica, las opciones políticas, el potencial militar, la economía y finanzas de la defensa, la cooperación industrial, la cultura de defensa o la gestión del personal civil y militar adscrito al Ministerio de Defensa); todo parece indicar que este proceso tiene un alcance más restringido y circunscrito a la esfera militar, pues a pesar de los importantes desarrollos legislativos que se están produciendo y que difícilmente pueden calificarse como transformadores, la Transformación parece haber sido planteada como la continuación lógica del proceso de modernización material, de la profesionalización de las fuerzas armadas, de la adaptación de la arquitectura defensiva del país a la caída de la Unión Soviética y del largo recorrido para integrar a España en las estructuras de seguridad y defensa colectivas ${ }^{4}$.

Han pasado ya ocho años desde que el proceso de Transformación pasara a vertebrar formalmente el planeamiento de la defensa español; y a pesar de que se han realizado significativos avances en la configuración normativa, orgánica, funcional y doctrinal de las fuerzas armadas, en la actualidad este proceso parece hallarse lastrado por la indefinición de los objetivos a lograr, la escasez de expertos que orienten este proceso, una inadecuada gestión de los principales programas de obtención de armamento y material o la falta de ambición para emprender profundos cambios en la estructura de fuerzas y catálogo de capacidades militares para incrementar la operatividad de los ejércitos y adaptar la arquitectura de defensa nacional a los múltiples retos del siglo XXI.

Teniendo estos elementos en cuenta, a continuación se pasará a realizar un somero análisis de la marcha del proceso de Transformación español desde sus orígenes hasta nuestros días.

\footnotetext{
${ }^{4}$ Para conocer la historia militar reciente de nuestro país es esencial la lectura de la siguiente obra: Ortega, Jorge (2008): La transformación de los ejércitos españoles (1975-2008), Madrid, Universidad Nacional de Educación a Distancia.
} 


\section{La Transformación militar en España}

Aunque formalmente la Transformación militar española arrancó en el año 2004, sus fundamentos fueron establecidos por la Revisión Estratégica de la Defensa que vio la luz un año antes ${ }^{5}$. Concebido como un exhaustivo estudio del entorno global y de los riesgos que se cernían sobre España con objeto de construir los pilares de la política de defensa y militar del país para el siglo $\mathrm{XXI}^{6}$, este documento definía el modelo futuro de fuerzas armadas, establecía la estructura de fuerzas y el catálogo de capacidades militares a desarrollar y trazaba las pautas de evolución a medio plazo con el objeto de satisfacer los cometidos presentes y futuros de la defensa nacional. Con este documento, nuestro país sentaba las bases para emprender el proceso de Transformación militar siguiendo de cerca los pasos iniciados por Estados Unidos en el año 2001 y la Alianza Atlántica en $2002^{7}$.

En este sentido, el trabajo comenzaba avalando las cifras propuestas en el año 1999 por la Ley 17/1999, del 18 de Mayo, del Régimen de Personal de las Fuerzas Armadas y proponía un objetivo de fuerzas para el ejército español comprendido entre 102.000 y 120.000 soldados y marineros (sumando a los cuadros de mando, esta cifra podría ascender a 170.000 efectivos $)^{9}$. Con este volumen de fuerzas, se pretendía disponer de un Ejército de Tierra que no sólo fuera capaz de garantizar la defensa del territorio nacional y mantener la presencia avanzada en los territorios extrapeninsulares; sino también participar en dos operaciones de gestión de crisis con dos fuerzas de entidad Brigada o en una sola operación con un cuartel general divisionario capaz de liderar una fuerza multinacional y dos Brigadas, e incluso participar en labores de defensa colectiva o respuesta a crisis con un cuartel general de Cuerpo de Ejército capaz de activar un mando componente terrestre multinacional y una División. En el caso de la Armada, ésta debería garantizar la defensa y el control de las aguas nacionales y estar en condiciones de participar en operaciones de gestión de crisis liderando un componente naval multinacional participando con un grupo de proyección aeronaval y anfibio, operar en dos escenarios distintos con dos escoltas en cada uno de ellos de manera continuada, mantener permanentemente dos cazaminas en una fuerza multinacional de lucha contraminas y desplegar dos submarinos a escenarios distintos. Finalmente, en el caso del Ejército del Aire, además de garantizar la defensa del espacio aéreo nacional, éste debería estar capacitado para desplegar dos escuadrones de combate en dos escenarios distintos y apoyar las operaciones en un tercero, garantizar el despliegue y sostenimiento estratégico de

\footnotetext{
${ }^{5}$ Ministerio de Defensa (2003): Revisión Estratégica de la Defensa, Madrid, Secretaría General Técnica, en www.mde.es/Galerias/docs/politica/seguridad-defensa/DGL_RevisionEstrategica.pdf.

${ }^{6}$ Un heterodoxo pero interesante análisis sobre la evolución de la situación estratégica de nuestro país puede hallarse en: Pérez, Jesús M. (2010): Guerras Posmodernas, Barcelona, El Cobre, pp. 81-99.

${ }^{7}$ Durante la Cumbre de Praga de 2002, los Jefes de Estado y de Gobierno aliados acordaron emprender la Transformación militar de la OTAN con objeto de desarrollar las herramientas necesarias para hacer frente a las "nuevas y graves amenazas y los profundos retos para la seguridad del siglo XXI". Para ello, este proceso se fundamentaría en tres iniciativas: la definición de una nueva estructura de mando; un nuevo diseño de fuerzas que culminaría con la creación de la NATO Response Force (NRF) y la potenciación del catálogo de capacidades militares. Organización del Tratado del Atlántico Norte (2003): The Prague Summit and NATO's Transformation, Bruselas, NATO Public Diplomacy Division.

${ }^{8}$ Ley 17/1999, del 18 de Mayo, del Régimen de Personal de las Fuerzas Armadas. Boletín Oficial del Estado, 19 de Mayo de 1999, núm. 119, p. 18751.

${ }^{9}$ Estos objetivos de fuerza pronto fueron reducidos - tal y como recogió la Ley 39/2007, del 19 de Noviembre, de la Carrera Militar - a unos más realistas 130.000 efectivos, compuestos por 50.000 cuadros de mando y 80.000-90.000 soldados y marineros. No obstante, la crisis económica que está sufriendo España obligará a revisar a la baja estos objetivos de fuerza. González, Miguel: "El ajuste obliga a las Fuerzas Armadas a prescindir de 6.000 soldados", El País, 30 de mayo de 2010, en

www.elpais.com/articulo/espana/ajuste/obliga/Fuerzas/Armadas/prescindir/6000/soldados/elpepunac/20100530e lpepinac_15/Tes.
} 
la fuerza y contribuir activamente a la iniciativa de Defensa Aérea Integrada Europea de la OTAN $^{10}$.

Y para satisfacer este conjunto de cometidos en una coyuntura marcada por una Revolución en los Asuntos Militares que prometía transformar el arte de la guerra y la proliferación de nuevos riesgos y amenazas a la seguridad internacional, la Revisión Estratégica de la Defensa definía un nuevo modelo de fuerzas armadas fundamentado en las siguientes características:

- Movilidad y capacidad de proyección para garantizar el rápido y eficaz despliegue estratégico de la fuerza y asegurar su movilidad táctica en el teatro de operaciones.

- Sostenibilidad en escenarios lejanos gracias a una logística integrada y unos relevos de fuerza adecuados.

- Disponibilidad mediante un adiestramiento y equipamiento permanentes y un nivel de alistamiento adecuado a los cometidos a desempeñar.

- Superioridad en el enfrentamiento mediante el empleo de armamento tecnológicamente avanzado.

- Protección de la fuerza frente a ataques realizados con convencional como noconvencional.

- Mando y control integrado para lograr la superioridad en la decisión ${ }^{11}$.

- Persistencia en la vigilancia, reconocimiento, inteligencia y adquisición de objetivos para lograr la superioridad en la información del entorno operativo.

- Modularidad e interoperabilidad de la fuerza para constituir ad-hoc Fuerzas Operativas Conjunto Combinadas capaces de enfrentarse a cualquier contingencia ${ }^{12}$.

- Acción conjunta para desempeñar con eficacia cualquier operación.

- Importancia de las fuerzas especiales para combatir con eficacia contra cualquier adversario convencional, irregular o híbrido.

\footnotetext{
${ }^{10}$ Marquina, Antonio: "La Revisión Estratégica de la Defensa: las misiones de las Fuerzas Armadas, las Fuerzas Armadas del Siglo XXI", UNISCI Discussion Papers, n 4 (enero 2004), pp. 1-9, en www.ucm.es/info/unisci/revistas/rev2.pdf.

${ }^{11}$ Como puede constatarse, estas seis primeras características son muy similares a las cinco grandes áreas de capacidad que definió la Alianza Atlántica en su Iniciativa de Capacidades de Defensa de 1999, que constituye el antecedente directo del Compromiso de Capacidades de Praga de 2003. Para más información, véase: Flowers, James N. (2002): NATO's Defense Capabilities: a Strategic Analysis, Carlisle Barracks, U.S. Army Strategic Studies Institute, en www.handle.dtic.mil/100.2/ADA401880.

${ }^{12}$ El concepto de Fuerza Operativa Conjunto Combinada (Combined Joint Task Force - CJTF) fue adoptado por la OTAN en la Cumbre de Bruselas (1994) para dotar a la Alianza de una fuerza capaz de enfrentarse a una amplia gama de contingencias mientras facilitaba el empleo de recursos y capacidades militares aliadas por parte de la Unión Europea Occidental en el marco de la nueva Iniciativa Europea de Seguridad y Defensa. En este sentido, las CJTF son “...fuerzas operativas multinacionales (combinadas) y de diferentes armas y servicios (conjuntas), organizadas de modo funcional para poder cubrir toda la gama de misiones militares de la Alianza que requieran el mando y control multinacional e inter-ejércitos de un Cuartel General de CJTF." Organización del Tratado del Atlántico Norte (2001): Manual de la OTAN, Bruselas, Oficina de Información y Prensa, p. 268.
} 
- Capacidad de operar en red para incrementar la coordinación, rapidez, eficiencia, precisión y seguridad de las operaciones.

Los cometidos identificados y el catálogo de capacidades propuestos sentaban las bases para la definición del modelo futuro de fuerzas armadas y los ejes sobre los que se articularía la Transformación militar española, un proceso que debería priorizar la acción conjunta y combinada, la capacidad expedicionaria y el dominio de todo el espectro operativo para convertir al Ejército de Tierra en una fuerza modular dotada de una gran flexibilidad operativa, una importante capacidad de proyección, una elevada movilidad táctica y una adecuada capacidad resolutiva; a la Armada en una fuerza capaz de proyectar su poder en la región litoral y tierra adentro; y al Ejército del Aire en una fuerza dotada con modernos sistemas de ataque de precisión a gran distancia, avanzados medios de transporte aéreo para garantizar la rápida proyección de la fuerza, vehículos aéreos no-tripulados y plataformas espaciales $^{13}$.

En conclusión, fundamentada en unos cometidos muy ambiciosos y difícilmente realizables con los recursos, medios y capacidades militares previsibles a medio plazo, estrechamente ligada al proceso de modernización de armamento y material ${ }^{14}$ y sin una planificación económica realista que garantizara la viabilidad de los proyectos en curso, la Revisión Estratégica de la Defensa - un trabajo que contó con la participación de la magra comunidad de defensa española y logró la bendición de los dos grandes partidos políticos del país - tuvo un limitado impacto en la definición de la política de defensa nacional. Y es que su tardía presentación en una legislatura agotada a mediados de 2003 tras un trágico accidente aéreo en el que fallecieron sesenta y dos militares españoles que regresaban de Afganistán, imposibilitó su puesta en práctica; y el cambio de Gobierno relegó al olvido esta completa declaración de intenciones que debía guiar el proceso Transformación militar español ${ }^{15}$. Sin embargo, el grueso de los objetivos, propuestas y planteamientos contenidos en este documento continúan constituyendo - al menos formalmente - los ejes sobre los que se articula la política de defensa y militar de nuestro país.

En el año 2004, la Directiva de Defensa Nacional 1/2004, del 30 de Diciembre, estableció definitivamente la Transformación de las fuerzas armadas como una de las principales líneas de actuación del Ministerio de Defensa para los años venideros. Este documento elaborado durante el primer año de cada legislatura con el fin de perfilar las directrices básicas de la política de defensa y militar del país, identificaba la necesidad de impulsar el proceso de Transformación para acomodar la arquitectura de seguridad y defensa

\footnotetext{
${ }^{13}$ Una visión complementaria sobre los factores que deberían guiar el proceso de Transformación militar español en sus inicios puede hallarse en: Cosidó, Ignacio y Bardají, Rafael L.: "La Transformación de las Fuerzas Armadas", Fundación para el Análisis y los Estudios Sociales (noviembre 2003), en www.gees.org/articulo/322.

${ }^{14}$ En efecto, la Revisión Estratégica de la Defensa parecía vincular la obtención del nuevo catálogo de capacidades militares con la entrada en servicio de los grandes programas de material en curso: los carros Leopardo, los vehículos de combate de infantería Pizarro, el Sistema Integrado de Artillería de Campaña (SIAC), el helicóptero de transporte NH-90 y el helicóptero de ataque Tigre en el Ejército de Tierra; las fragatas F-100, los submarinos S-80, los Buques de Acción Marítima (BAM) y el Buque de Proyección Estratégica (BPE) en la Armada, y el avión de caza EF-2000 y el avión de transporte A-400M en el Ejército del Aire.

${ }^{15}$ Es interesante comentar que un subproducto de la Revisión Estratégica de la Defensa fue la Estrategia Militar Española, un trabajo que tomaba como base las ideas recogidas en la primera para plantear la estructura de fuerzas, el catálogo de capacidades, el marco para la actuación conjunta y las grandes líneas para el planeamiento estratégico y operacional del Jefe de Estado Mayor de la Defensa (Estado Mayor de la Defensa (2003): Nuevos retos, nuevas respuestas: estrategia militar española, Madrid, Secretaría General Técnica).
} 
española a un mundo en constante evolución. Para ello, el Gobierno no sólo determinaría durante la legislatura 2004-2008 - un nuevo nivel de ambición militar, definiría el modelo futuro de fuerzas armadas y propondría una entidad de fuerza adecuada a los cometidos a desempeñar; sino también emprendería la Transformación militar mediante la adquisición de un nuevo catálogo de capacidades militares y la reestructuración de las fuerzas existentes con objeto de incrementar su movilidad estratégica y táctica, flexibilidad, modularidad, interoperabilidad y capacidad de acción conjunta.

Y para garantizar la viabilidad económica de la Transformación, un proceso que se armonizaría con las iniciativas realizadas por la Alianza Atlántica y la Unión Europea, el trabajo manifestaba la determinación del Gobierno por lograr la estabilidad presupuestaria necesaria para financiar la adquisición de los sofisticados materiales que parecían constituir el eje de este proceso ${ }^{16}$.

En resumen, la Directiva de Defensa Nacional 1/2004 marcó el comienzo formal de la Transformación militar española y su emplazamiento como uno de los ejes que articularían la política de defensa y militar del país para los años venideros. No obstante, algunas importantes iniciativas propuestas en este documento programático no fueron finalmente implementadas - caso de la elaboración de una nueva estrategia militar o la determinación de un nuevo nivel de ambición para las fuerzas armadas - por lo que tanto los objetivos estratégicos, los niveles de esfuerzo como el volumen de fuerzas continuaron fundamentándose formalmente en los preceptos establecidos en la Revisión Estratégica de la Defensa y en la Estrategia Militar Española de $2003^{17}$.

Igualmente, aunque se lograron los objetivos de gasto previstos, este incremento en el presupuesto de defensa no comportó ninguna mejora en la eficiencia económica del Ministerio susceptible de garantizar la financiación, presente y futura, del proceso de Transformación. Muchos países de nuestro entorno estaban inmersos en una Revolución en los Asuntos de Negocios (Revolution in Business Affairs) ${ }^{18}$ que, fundamentada en la racionalización de los procesos de adquisición de armamento y material, la flexibilización de la gestión de los programas, la reforma de las técnicas contables, la reducción de redundancias o la externalización de funciones, pretendía garantizar la viabilidad de la Transformación mediante la optimización de los recursos disponibles, la reducción de los gastos corrientes, la cancelación de los programas obsoletos y la liberación de fondos para la adquisición de nuevos sistemas más relevantes para los conflictos actuales.

Sin embargo, la gestión de la defensa de nuestro país no solo continuó anclada en las prácticas financieras, burocráticas y empresariales tradicionales, y los principales programas de obtención de armamento y material no fueron sometidos a ninguna revisión externa e independiente sobre su marcha, empleabilidad o conveniencia actual y futura ${ }^{19}$; sino que su

\footnotetext{
${ }^{16}$ Un análisis más completo de este documento puede hallarse en: Arteaga, Félix: "La Directiva de Defensa Nacional 1/2004", Análisis del Real Instituto Elcano, no 29 (febrero de 2005), s. n., en Www.realinstitutoelcano.org/analisis/692/Arteaga\%20pdf.pdf.

${ }^{17}$ Arteaga, Félix y Fojón, Enrique (2008): El planeamiento de la política de defensa y seguridad en España, Madrid, Instituto Universitario General Gutiérrez Mellado, pp. 355-59.

${ }^{18}$ Lippitz, Michael; O'Keefe, Sean and While, John (eds.) (2008): Keeping the Edge: Managing Defense for the Future, Cambridge, Preventive Defense Project, en www.belfercenter.ksg.harvard.edu/ publication/1799/keeping the edge.html.

${ }^{19}$ Sin contar con los efectos de la crisis económica sobre el presupuesto de defensa presente y futuro, estos grandes programas han comprometido aproximadamente el $80 \%$ de los recursos previsibles hasta el año 2020 (Arteaga y Fojón, op. cit., pp. 383-384). Ello pone en una difícil situación al Ministerio de Defensa pues, si no
} 
financiación continuó fundamentada en el sistema de pagos aplazados con anticipos a cargo del Ministerio de Industria. Este modelo se planteó a mediados de los noventa como una solución eventual para garantizar la adquisición de nuevo material militar en un periodo de crisis económica y debía haber sido reemplazado tan pronto aumentara la liquidez del Ministerio de Defensa ${ }^{20}$. No obstante, esta fórmula se ha mantenido intacta hasta fecha de hoy, a pesar de haber disfrutado de una época de bonanza económica y de los cambios realizados en las fórmulas de contratación pública. Ello no solo ha lastrado, indefectiblemente, nuestra Transformación militar; sino que también es una de las principales responsables de la inviabilidad económica de nuestra programación de la defensa ${ }^{21}$ que, fundamentada en la adquisición de grandes programas, se halla en una delicada situación debido a una deuda que asciende a más de 27.000 millones de euros ${ }^{22}$.

Después de que la Directiva de Defensa Nacional 1/2004 sentara las bases para la Transformación, en el ámbito normativo se emprendieron los cambios necesarios para llevarla a cabo. El primero fue la promulgación de la Orden de Defensa 1076/2005, del 19 de Abril, por la que se desarrolla la estructura del Estado Mayor de la Defensa ${ }^{23}$. Con ella no sólo se configuraba un Estado Mayor de la Defensa que disponía de todos los elementos necesarios para planear y conducir operaciones conjuntas (un mando conjunto de operaciones y un centro de inteligencia militar conjunta); sino también un pequeño centro de Transformación que, “...responsable de la investigación y estudio de nuevos conceptos organizativos y de doctrina, así como de la implantación de nuevas tecnologías”, intentaría armonizar las iniciativas aliadas, europeas o multilaterales en materia de Transformación y promover el desarrollo de nuevas capacidades militares adecuadas a los retos actuales y futuros. Igualmente, este centro debería constituirse como un foro de reflexión para modernizar el pensamiento militar español con objeto de adecuarlo a un escenario táctico, operativo y estratégico de gran complejidad y en constante evolución ${ }^{24}$.

Desde su constitución, este centro no sólo ha sido el responsable de coordinar el desarrollo de la doctrina conjunta nacional y conjunto-combinada aliada, participar en proyectos bilaterales de Transformación con países como Estados Unidos, Francia, Alemania, Reino Unido o Finlandia, multilaterales mediante la colaboración en la serie de Experimentos

realizan con urgencia cambios en la programación militar, nuestras fuerzas armadas difícilmente dispondrán de los recursos necesarios para afrontar los cambios tecnológicos o satisfacer los nuevos requerimientos operativos.

${ }^{20}$ Pérez, Francisco (2009): El gasto en Defensa en España 1946-2009, Madrid, Publicaciones del Ministerio de Defensa, pp. 325-348.

${ }^{21}$ Mientras se está redactando este trabajo, España está negociando la venta de doscientos carros Leopard $2 E$ a Arabia Saudita. Aunque se desconocen los detalles de la negociación (en particular, cuántos carros procederán de los lotes originalmente destinados a nuestro Ejército de Tierra), esta iniciativa realizada en un contexto de crisis económica parece constituir el reconocimiento tácito del excesivo volumen de carros inicialmente propuesto (212 Leopard 2E y 108 Leopard 2A4), de la urgencia de liberar fondos para la compra de otros equipos que satisfagan los requerimientos operativos presentes y de la mala planificación de las adquisiciones. González, Miguel, "España negocia la venta de más de 200 carros de combate a Arabia Saudî", El País, 25 de Octubre de 2010, en

www.elpais.com

/articulo/espana/Espana/negocia/venta/200/carros/combate/Arabia/Saudi/elpepuesp/20101025elpepinac 5/Tes.

${ }^{22}$ González, Miguel: "Defensa renegocia una deuda de 26.000 millones que no puede pagar", El País, 12 de Agosto de 2011, en

www.politica.elpais.com/politica/2011/08/12/actualidad/1313163003 049342.html.

23 Orden de Defensa 1076/2005, del 19 de Abril, por la que se desarrolla la estructura del Estado Mayor de la Defensa. Boletín Oficial del Estado, 26 de Abril de 2005, núm. 99, p. 14049. La estructura aquí planteada será definida con mayor detalle en la Instrucción 40/2008, del 15 de Abril, del Jefe de Estado Mayor de la Defensa, sobre organización del Estado Mayor de la Defensa. Boletín Oficial de Defensa, 29 de Abril de 2008, núm. 84, p. 5595.

${ }^{24}$ Arteaga y Fojón, op. cit., p. 367. 
Multinacionales $^{25}$ o con la OTAN a través del Mando Aliado de Transformación; sino también de implementar en nuestro país el proceso de desarrollo y experimentación de conceptos para apoyar el diseño de nuevas capacidades militares (entre los que destacan el Desarrollo de Conocimiento, la Concienciación Transcultural, la Comunicación Estratégica, las Operaciones Multinacionales de Información o el Conocimiento del Entorno Marítimo), liderar el primer estudio prospectivo para informar sobre la evolución del entorno estratégico y guiar el proceso de Transformación militar ${ }^{26}$ o definir un nuevo Enfoque Integral a la seguridad, a la gestión de crisis complejas y a las operaciones de estabilización ${ }^{27}$, entre otras numerosas iniciativas encaminadas a preparar las fuerzas armadas españolas para satisfacer los retos presentes y futuros ${ }^{28}$.

No obstante, a pesar de estos éxitos que permiten avanzar en la Transformación militar del país, no parece que a fecha de hoy este centro haya conseguido crear una masa crítica de expertos militares y civiles suficientemente numerosos, preparados e influyentes para impulsar la Transformación, transmitir a todas las esferas de la defensa española la trascendencia de este proceso (una realidad un tanto paradójica cuando este proceso no sólo constituye una de las líneas generales de actuación de la defensa nacional, sino también es un asunto recurrente en las declaraciones públicas de las altas autoridades políticas y militares del Ministerio de Defensa), asegurar la consolidación de los avances realizados o implantar firmemente estos cambios en la cultura de las fuerzas armadas; por lo que estos asuntos deberán constituir una de las máximas prioridades de este y de otros organismos asociados o vinculados con este proceso a lo largo de los próximos años.

La segunda reforma se produjo con la entrada en vigor de la Orden Ministerial 37/2005, del 30 de Marzo, por la que se regula el proceso de Planeamiento de la Defensa ${ }^{29}$ e incidía sobre el sistema de definición de objetivos, establecimiento de prioridades, asignación de recursos y ejecución de las actividades requeridas para generar una estructura de fuerzas y un catálogo de capacidades adecuado para satisfacer los objetivos de seguridad y defensa marcados por la Directiva de Defensa Nacional en curso ${ }^{30}$.

La entrada en vigor de esta nueva norma supuso la derogación de la Directiva 38/1996, del 27 de Febrero, por la que se regula el proceso de planeamiento de la Defensa Militar, y con ella se abandonaba el planeamiento militar tradicional basado en amenazas - empleado para contrarrestar un adversario concreto del que se conocen sus capacidades militares, el modo en que emplea sus fuerzas y los escenarios donde podría combatir - y se sustituía por un nuevo sistema de planeamiento militar que, considerado como uno de los ejes de la

\footnotetext{
${ }^{25}$ Hasta la fecha, España ha participado en el Multinational Experiment 5 (MNE5), un proyecto que pretendía avanzar en la cooperación multinacional e interagencias en operaciones de estabilización bajo el marco de un Enfoque Integral (Comprehensive Approach); y en el MNE6 para proponer el empleo de un Enfoque Integral para combatir contra adversarios irregulares. Fojón, Enrique: "Los experimentos multinacionales, un camino a la transformación”, Revista Española de Defensa nº 238 (febrero 2008), pp. 30-32.

${ }^{26}$ Barcenas, Luis: "Primer 'estudio de futuros' para las Fuerzas Armadas", Atenea Seguridad y Defensa, 19 de diciembre de 2009; en

www.revistatenea.es/RevistaAtenea/REVISTA/articulos/Gestion Noticias_1349_ESP.asp.

27 Pareja, Iñigo y Colom, Guillem: "El Enfoque Integral (Comprehensive Approach) a la gestión de crisis internacionales", Análisis Real Instituto Elcano, n 115 (septiembre 2008), s.n., en

www.realinstitutoelcano.org/ARI115-2008_Colom_Piella_Enfoque_Integral.pdf.

${ }_{28}$ Un análisis más amplio de la estructura, cometidos, iniciativas e hitos de este centro de transformación puede hallarse en www.mde.es/Galerias/ooee/fichero/EMD_Unidad_UTRAFAS.pdf.

${ }^{29}$ Orden Ministerial 37/2005, del 30 de Marzo, por la que se regula el proceso de Planeamiento de la Defensa. Boletín Oficial de Defensa, 8 de Abril de 2005, núm. 68, p. 3579.

${ }^{30}$ Colom, Guillem: "El proceso de planeamiento de la Defensa Nacional", Revista General de Marina vol. 206, $n^{\circ} 5$ (Junio 2011), pp. 833-842.
} 
Transformación y adoptado por nuestros socios europeos y aliados, se orienta a la obtención de un catálogo de capacidades polivalentes, flexibles, equilibradas, asequibles y susceptibles de ser empleadas en toda la gama de escenarios presentes y futuros ${ }^{31}$.

En el caso español, cada ciclo de planeamiento tiene una duración de cuatro años (dos años de ejecución y dos años de revisión con el objeto de armonizarlo con el sistema de planeamiento OTAN); se realiza sobre tres horizontes temporales distintos (a largo plazo para orientar la transformación de la fuerza, a medio plazo para programar el empleo de los recursos; y a corto plazo para fijar las necesidades presupuestarias y ajustar la programación de los recursos) y se ejecuta en torno a dos procesos paralelos:

- El planeamiento militar para detallar el catálogo de capacidades, los requisitos operativos, la disponibilidad de la fuerza y los niveles de alistamiento requeridos para satisfacer nuestros objetivos de defensa. Este proceso está compuesto por los planeamientos de fuerzas y operativo, siendo el responsable de su confección y ejecución el Jefe de Estado Mayor de la Defensa.

- El planeamiento de recursos para lograr el Objetivo de Capacidades Militares en sus aspectos materiales, humanos y financieros. Mientras el secretario de Estado de Defensa ostenta la responsabilidad sobre los planeamientos de recursos financieros y materiales (determinación de los marcos financiero, tecnológico e industrial de la defensa y dirección de la obtención y sostenimiento del armamento y material, infraestructuras y sistemas de información y comunicaciones); el subsecretario de Defensa se responsabiliza del planeamiento de los recursos humanos (determinación del marco cualitativo y cuantitativo del personal y dirección en la obtención, formación y calidad de vida del mismo).

Hasta la fecha se ha realizado un ciclo completo de planeamiento (2005-2008) y está en marcha un segundo ciclo que finalizará en el año 2012. Éste arrancó a mediados de 2009 con la publicación de la Directiva de Política de Defensa 1/2009, del 5 de Mayo ${ }^{32}$ y tratará formalmente de profundizar en la acción conjunta entre los tres ejércitos, la combinada entre fuerzas de distintos países y la interagencias entre actores militares y civiles; adaptarse a la coyuntura económica optimizando los recursos disponibles e integrar los procesos de planeamiento aliado y europeo dentro del planeamiento nacional ${ }^{33}$.

\footnotetext{
${ }^{31}$ Aunque el planteamiento por capacidades no es en absoluto novedoso - fue originariamente empleado durante la década de los sesenta para apoyar la definición de la estrategia nuclear estadounidense y en la inmediata posguerra fría volvió a resurgir con la divulgación de la RMA - alcanzó fama mundial en el año 2001 cuando Donald Rumsfeld lo estableció como el estilo de planeamiento transformador. Y es que el titular del Pentágono no sólo entendía que el planeamiento tradicional era inadecuado para satisfacer las heterogéneas labores que llevaban a cabo las fuerzas armadas del país e inútil para afrontar los retos del nuevo siglo, sino que creía que la RMA estaba produciendo un catálogo de capacidades polivalentes y susceptibles de cubrir toda la gama de amenazas. (Davis, Paul K. y Finch, Lou (1993): Defense Planning for the Post-Cold War Era: Giving Meaning to Flexibility, Adaptiveness, and Robustness of Capability, Santa Monica, RAND Corporation).

${ }^{32}$ Al tratarse de un documento clasificado, la Directiva de Política de Defensa 1/2009 no puede ser consultada. Sin embargo, un resumen ejecutivo de la misma puede hallarse en www.mde.es/Galerias /politica/seguridaddefensa/ficheros/DGL_ResumenEjecutivDPD 1-2009.pdf.

${ }^{33}$ Los objetivos propuestos para el ciclo de planeamiento en curso son los siguientes: (1) garantizar la defensa nacional y salvaguardar los intereses nacionales, tanto en el marco de la defensa colectiva como de manera autónoma; (2) contribuir a la paz y seguridad internacional a través de las Naciones Unidas, la Unión Europea, la Alianza Atlántica, la Organización para la Seguridad y la Cooperación en Europa u otras iniciativas de
} 
El catálogo de capacidades militares que articula el planeamiento de la defensa español se divide en seis grandes áreas ${ }^{34}$ que, a su vez, constituyen las grandes líneas de la Transformación militar nacional. Y como podrá constatarse, éstas guardan enormes similitudes con las características definidoras del modelo futuro de fuerzas armadas propuesto en el año 2003 por la Revisión Estratégica de la Defensa y con los ejes de la Transformación militar de la Alianza Atlántica ${ }^{35}$ :

- Mando y control integrado mediante la adquisición de sistemas de mando, control y comunicaciones que enlacen desde el nivel estratégico al táctico y permitan lograr la superioridad en la información y en la toma de decisiones.

- Superioridad en el enfrentamiento mediante la adquisición de modernos medios que permitan garantizar la libertad de acción de la fuerza y proporcionar una amplia gama de respuestas que permitan cumplir con las misiones encomendadas.

- Vigilancia, reconocimiento, inteligencia y adquisición de objetivos (ISTAR) mediante la obtención de sistemas integrados y tecnológicamente avanzados para lograr la superioridad en la información y en la decisión.

- Movilidad y capacidad de proyección mediante la adquisición de medios que garanticen la proyección estratégica y la movilidad táctica de la fuerza para aplicar el poder militar donde y cuando sea necesario.

- Sostenibilidad mediante la coordinación del apoyo logísticos de los tres ejércitos, la eficaz organización y ejecución del transporte estratégico y la coordinación con otros organismos civiles y militares para así garantizar el sostenimiento de la fuerza desplegada en zona de operaciones.

- Supervivencia y protección mediante el uso de recursos, equipos e infraestructuras que reduzcan los niveles de riesgo de las fuerzas propias frente a cualquier amenaza previsible y la supervivencia de las unidades frente a ataques enemigos.

Aunque este nuevo sistema por capacidades permitirá armonizar los ciclos de planeamiento español con el aliado, sistematizar la Transformación y dotar a nuestras fuerzas armadas de un catálogo de capacidades polivalentes, flexibles y susceptibles de ser empleadas en una amplia gama de escenarios; lo cierto es que hasta fecha de hoy este sistema parece haberse usado

naturaleza multilateral; (3) continuar impulsando el desarrollo de la Política Europea de Seguridad y Defensa; (4) intensificar la diplomacia de defensa con aquellos países y regiones de interés estratégico para España; (5) promover el desarrollo de mecanismos que permitan integrar los esfuerzos civiles y militares, tanto públicos como privados, para la seguridad y defensa nacional; (6) mantener el impulso al proceso de Transformación y consolidar los avances producidos en el proceso de modernización de los ejércitos; (7) afianzar el modelo profesional de las fuerzas armadas desarrollando la Ley de la Carrera Militar; (8) consolidar el proceso de planeamiento de la defensa por capacidades; fomentar la eficiencia en la gestión de los recursos asignados a la defensa y desarrollar la estrategia de sostenimiento del ciclo de vida de las capacidades; (9) potenciar la dimensión social y cultural de la defensa; y finalmente (10) desarrollar un marco financiero estable que permita alcanzar los anteriores objetivos.

${ }^{34}$ Estado Mayor de la Defensa (2008): Fuerzas Armadas españolas: mejorando la eficacia operativa, Madrid, Ministerio de Defensa, pp. 6-7, en www.mde.es/Galerias/ooee/fichero/EMD_FAS_Mejorando.pdf.

35 NATO Strategic Commanders (2004): Strategic Vision, the Military Challenge, Bruselas, NATO Public Information Office. 
para apoyar la obtención de unos grandes programas en curso que, heredados tanto de la Guerra Fría como de los gobiernos anteriores ${ }^{36}$, apenas satisfacen las necesidades presentes pero que han hipotecado las cuentas presentes y futuras del Ministerio de Defensa ${ }^{37}$; a la vez que se ha demostrado incapaz de prever, priorizar o financiar la adquisición y sostenimiento de otros sistemas más convenientes para los conflictos actuales. En este sentido, la compra por procedimiento de urgencia de los vehículos LMV Lince y los vehículos contra minas y emboscadas MRAP RG-31Mk5 - en gran parte motivada la presión de la opinión pública ${ }^{38}$ parece haberse realizado al margen de este proceso sin haber ganado flexibilidad (la adquisición, recepción, preparación y despliegue de estos sistemas se ha demorado durante casi dos años en el caso del RG-31Mk5) ${ }^{39}$ y obviando el sostenimiento y el ciclo de vida de estos vehículos ${ }^{40}$.

En otras palabras, aunque el sistema de planeamiento por capacidades ofrece infinitas ventajas respecto al estilo tradicional, será también necesario que en los sucesivos ciclos de planeamiento sus responsables reevalúen las prioridades de adquisición y opten por la obtención de equipos verdaderamente transformadores en detrimento de los sistemas heredados, cuya cancelación o rebaja en las opciones de compra permitiría liberar unos preciados recursos esenciales para avanzar en el proceso de Transformación militar o para satisfacer las necesidades operativas observadas por las fuerzas desplegadas en el exterior ${ }^{41}$.

La tercera y última gran reforma para la Transformación fue la promulgación de la Ley Orgánica 5/2005, del 17 de Noviembre, de la Defensa Nacional ${ }^{42}$, que sustituía a la obsoleta norma de $1984^{43}$. Esta ley pretendía acomodar los criterios básicos de la defensa nacional y de

\footnotetext{
${ }^{36}$ El carro Leopardo, el vehículo de combate de infantería Pizarro, el Sistema Integrado de Artillería de Campaña, el helicóptero de transporte NH-90 y el helicóptero de ataque Tigre en el Ejército de Tierra; las fragatas F-100, los submarinos S-80, los Buques de Acción Marítima y el Buque de Proyección Estratégica en la Armada, y los aviones de caza EF-2000 y el avión de transporte A-400M en el Ejército del Aire.

${ }^{37}$ En el marco de las XIV Jornadas de la Fundación Círculo de Tecnologías para la Defensa y la Seguridad (Madrid, 25 y 26 de Octubre de 2010), el secretario de Estado de Defensa afirmó que: "No deberíamos haber adquirido sistemas para escenarios que no existen y con un dinero que no teníamos." (A.V.S., "Constantino Méndez: La capacidad tecnológica de España no ha tenido reflejo en la gestión de programas", Infodefensa, 27 de Octubre de 2010, en

www.infodefensa.com/esp/noticias/noticias.asp?cod=2764\&n=Constantino-Méndez:-"La-capacidadtecnológica-de-España-no-ha -tenido-reflejo-en-la-gestión-de-programas".

${ }^{38}$ En efecto, el goteo de ataques en Afganistán y el Líbano contra los vetustos vehículos BMR provocó que los medios de comunicación españoles prestaran atención a este asunto y situaran la obsolescencia de estos vehículos en un lugar destacado de la agenda informativa nacional. Igualmente, es importante destacar el inusitado papel que jugó Internet en la toma de conciencia de esta realidad: "Blogueros de defensa se unen para exigir al Gobierno con el lema 〈La complacencia mata, ni una baja más`”, Europa Press, 10 de junio de 2008.

${ }^{39}$ Un duro análisis sobre esta situación puede hallarse en: Cortés, Hernando (seudo.): “Transformación y nuevos vehículos. En torno a los nuevos vehículos blindados especiales del Ejército de Tierra: LMV Lince y MRAP RG-31”, Grupo de Estudios Estratégicos, 27 de mayo de 2009, en

www.gees.org/articulos/transformacion_y_nuevos_vehiculos_en_torno_a_los_nuevos_vehiculos_blindados_esp eciales_del_ejercito_de tierra_lmv_lince_y_mrap_rg_31_6491.

${ }^{40}$ Nieves, Gema: "Defensa debe aprender a prever el mantenimiento de sus adquisiciones", Atenea Seguridad y Defensa, 2 de Noviembre de 2010; en www.revistatenea.es/RevistaAtenea/REVISTA/articulos/GestionNoticias 3253 ESP.asp.

${ }^{41}$ Colom, Guillem: "El proceso de planeamiento de la Defensa Nacional", Revista General de Marina Vol. 260, $\mathrm{n}^{\mathrm{o}} 5$ (junio 2011), pp. 833-42.

${ }^{42}$ Ley Orgánica 5/2005, del 17 de Noviembre, de la Defensa Nacional. Boletín Oficial del Estado, 18 de Noviembre de 2005, núm. 276, p. 37717.

${ }^{43}$ Ley Orgánica 1/1984, del 5 de Enero, de reforma de la Ley Orgánica 6/1980, del 1 de Julio, por la que se regulan los criterios básicos de la defensa nacional y la organización militar. Boletín Oficial del Estado, 7 de Enero de 1984, núm. 6, p. 389. Como puede constatarse, esta norma reformaba la Ley Orgánica 6/1980, del 1 de
} 
la organización militar a la realidad sociopolítica e internacional del siglo XXI mediante el establecimiento de un nuevo marco para la regulación, administración y gestión de la seguridad y la defensa nacional.

Entre las novedades contenidas por esta norma, cabe destacar la consideración de las fuerzas armadas como una entidad única orientada a la acción conjunta, la consolidación de la figura del Jefe de Estado Mayor de la Defensa, la organización de las fuerzas armadas en dos estructuras diferenciadas (una orgánica para la preparación de la fuerza y otra operativa para su empleo efectivo) ${ }^{44}$; la supeditación de la acción militar española a un mandato multilateral; la participación del Parlamento en la aprobación de los despliegues internacionales o la definición de un nuevo catálogo de misiones y una tipología de operaciones más amplio.

De esta forma, tal y como queda recogido en los artículos 15 y 16 de la Ley Orgánica de la Defensa Nacional, además de garantizar la soberanía e independencia de España, defender su integridad territorial y el ordenamiento constitucional mientras contribuyen militarmente a la seguridad y defensa de España y de sus aliados, al mantenimiento de la paz, la estabilidad y la ayuda humanitaria; las fuerzas armadas españolas también deben preservar la seguridad y el bienestar de los ciudadanos en los supuestos de grave riesgo, catástrofe, calamidad u otras necesidades públicas y la posible evacuación de los residentes españoles en el extranjero, cuando circunstancias de inestabilidad en un país pongan en grave riesgo su vida o sus intereses. Por lo tanto, el catálogo de operaciones a realizar por nuestras fuerzas armadas puede dividirse en la prevención de conflictos, la disuasión, el mantenimiento de la paz, las actuaciones en situación de crisis y la respuesta a agresiones.

La aprobación de esta norma ha sentado las bases para la elaboración de todo un conjunto de iniciativas legislativas - entre las que cabe destacar la Ley 8/2006, del 24 de Abril, de Tropa y Marinería, la Ley 39/2007, de 19 de Noviembre, de la carrera militar, el Real Decreto 1723/2008, de 24 de Octubre, por el que se crea el sistema de centros universitarios de la defensa, el Real Decreto 416/2006, del 11 de Abril, por el que se establece la organización y el despliegue del Ejército de Tierra, de la Armada y del Ejército del Aire, así como de la Unidad Militar de Emergencias ${ }^{45}$, o el Real Decreto 787/2007, del 15 de Junio, por el que se regula la estructura operativa de las Fuerzas Armadas, por poner los ejemplos más representativos - que han servido para adaptar los criterios básicos de la defensa nacional y la organización de las fuerzas armadas al mundo actual.

Sin embargo, estos desarrollos normativos no han estado exentos de controversias, pues la Ley de la Carrera Militar se ha topado con la oposición frontal de muchos oficiales y suboficiales que han visto truncadas sus expectativas de ascenso ${ }^{46}$, la Ley de Tropa y Marinería no parece solventar los problemas provocados por la profesionalización de las fuerzas armadas y la solución adoptada para integrar a los futuros oficiales dentro del Espacio Europeo de Educación Superior ha desaprovechado la histórica oportunidad de crear una

Julio, por la que se regulan los criterios básicos de la Defensa Nacional y la Organización Militar para garantizar el fin de la autonomía militar y la supremacía política al frente del Ministerio de Defensa.

${ }^{44}$ La organización y las relaciones funcionales entre ambas estructuras se consolidará con la entrada en vigor del Real Decreto 787/2007, de 15 de junio, por el que se regula la estructura operativa de las Fuerzas Armadas. Boletín Oficial del Estado, 16 de Junio de 2007, núm. 144, p. 26309

${ }^{45}$ Con la aprobación de este Real Decreto, la Unidad Militar de Emergencias - una fuerza creada, por acuerdo de Consejo de Ministros el 7 de Octubre de 2005, para intervenir dentro del territorio nacional en la gestión de desastres - se integraba dentro de la estructura militar como una fuerza conjunta de carácter permanente. Sin embargo, esta iniciativa difícilmente puede calificarse como transformadora.

${ }^{46}$ Cervilla, Paloma: "Los militares rompen su silencio sobre la polémica Ley de la Carrera Militar", $A B C, 29$ de Junio de 2009, en www.abc.es/20090629/nacional-politica/militares-rompen-silencio-sobre-20090629.html. 
Universidad Nacional de la Defensa capaz de realizar labores de enseñanza e investigación puntera en asuntos estratégicos 47 .

Todas estas iniciativas fueron avaladas, de facto, en la Directiva de Defensa Nacional 1/2008, del 30 de Diciembre, que fijaba las líneas generales de la política de defensa para la legislatura en curso (2008-2012). Este documento - el primero de estas características aprobado bajo el amparo de la Ley Orgánica 5/2005 de la Defensa Nacional - plantea la necesidad de continuar con la Transformación dinámica y permanente de nuestras fuerzas armadas con el fin de adaptarlas al cambiante entorno estratégico. Asimismo, es importante destacar el reconocimiento de la acción única del Estado en materia de seguridad y defensa siguiendo los preceptos del Enfoque Integral ${ }^{48}$ y el mandato por elaborar una Estrategia de Seguridad Nacional, un documento que, fundamentado “...en un profundo análisis de los intereses vitales españoles y en un sólido estudio de los riesgos y amenazas que nos afectan [...] permitirá la formulación de las líneas estratégicas de actuación."49.

Después de enormes retrasos en el calendario marcado, a mediados de 2011 se presentó la Estrategia Española de Seguridad: un compromiso de todos ${ }^{50}$. Aunque este trabajo es el primero de estas características elaborado en nuestro país, el producto resultante ha tenido una fría acogida debido a su indefinición estratégica, ambigüedad conceptual, imprecisión terminológica, incoherencia interna o discutible aplicabilidad práctica $^{51}$; y su impacto real será muy limitado, puesto que ha sido presentado al final de una legislatura que probablemente comportará un cambio de signo político y su contenido no ha sido consensuado con la oposición. En consecuencia, es muy probable que este documento no tenga ninguna utilidad práctica en la definición de las grandes líneas de la política de seguridad y defensa española ni tampoco en la Transformación militar del país

\section{Conclusiones}

En las páginas anteriores se ha realizado un breve repaso a la Transformación militar española, proceso que se inició formalmente en el año 2004 y que a fecha de hoy parece hallarse en un punto muerto. En efecto, en estos últimos seis años se han realizado algunos avances genéricos en varias áreas de capacidad (nuevas doctrinas conjuntas y específicas, una nueva estructura orgánica y operativa para el conjunto de las fuerzas armadas, nuevos

\footnotetext{
${ }^{47}$ La creación de esta Universidad Nacional de la Defensa era una iniciativa que estaba contemplada en los sucesivos borradores de la Ley de la Carrera Militar: "La Ley de la Carrera Militar contempla integrar las Academias Militares en la Universidad Nacional de la Defensa”, Europa Press, 14 de Febrero de 2006.

${ }^{48}$ Recuérdese que el concepto de Enfoque Integral (Comprehensive Approach) fue inicialmente propuesto por Gran Bretaña (Joint Doctrine \& Concepts Centre, Joint Discussion Note 4/05: The Comprehensive Approach, Ministry of Defense, Shrivenham, 2006), posteriormente adoptado, entre otros actores, por la Alianza Atlántica y la Unión Europea (Colom, Guillem (2009): "El Enfoque Integral aplicado a la Alianza Atlántica y la Unión Europea", en Los nuevos paradigmas de la seguridad, Madrid, Centro Internacional de Toledo para la Paz, pp. 39-47) y en nuestro país fue desarrollado por la unidad de Transformación de las fuerzas armadas.

49 Acuerdo del Consejo de Ministros por el que se ordena la elaboración de la Estrategia Española de Seguridad, 23 de Diciembre de 2009, en www.la-moncloa.es/ConsejodeMinistros/Referencias/_2009/refc20091223.htm\#Estrategia.

${ }_{50}$ Ministerio de la Presidencia (2011): Estrategia Española de Seguridad: una responsabilidad de todos, Madrid, Ministerio de la Presidencia, en www.lamoncloa.gob.es/NR/rdonlyres/9BD221CA-A32A-4773-ACB7ECD3FC 6C9B9E/0/ESTRATEGIAESPANOLADESEGURIDAD.pdf.

${ }^{51}$ Véase, por ejemplo: Alejandre, Luis: "Estrategia de Seguridad", La Razón, 14 de julio de 2011; Tafalla, Ángel: "Estrategia Española de Seguridad: preguntas sin respuesta", Atenea Seguridad y Defensa, 4 de Julio de 2011 o Mangas, Araceli: “La Estrategia Española de Seguridad”, El Mundo, 29 de junio de 2011.
} 
modelos de enseñanza y adiestramiento o nuevas políticas de personal), complementadas por un conjunto de normas, más o menos afortunadas, encaminadas a adaptar la reglamentación de la defensa nacional a los estándares actuales.

Sin embargo, la Transformación militar española se ha visto lastrada por unos objetivos inadecuados y obsoletos que se remontan a la Revisión Española de la Defensa de 2003, la escasez de expertos independientes capaces de orientar este proceso y evaluar críticamente las decisiones realizadas, la ausencia de debate político y académico serio sobre su definición e implementación, la carencia de un grupo numeroso e influyente que impulse el cambio dentro de las fuerzas armadas, la escasa conciencia de la comunidad de defensa española sobre la trascendencia estratégica que tiene, la falta de una cultura de Transformación, la falta de un liderazgo político-militar claro para implementarla y, finalmente, por una inadecuada gestión de los recursos disponibles, una discutible política industrial y el mantenimiento de unos programas de obtención de armamento y material que, en muchos casos, apenas satisfacen las necesidades presentes y futuras de las fuerzas armadas, pero que han hipotecado las cuentas del Ministerio de Defensa para los años venideros.

Este conjunto de elementos han lastrado la Transformación militar española y dificultarán la adaptación de nuestras fuerzas armadas al heterogéneo, fluido y cambiante campo de batalla futuro. Sería conveniente que en un futuro inmediato se establezcan los fundamentos normativos, conceptuales y estratégicos necesarios para trazar un plan de Transformación militar más ambicioso, realista y asequible, que entrañe la racionalización de la organización, funcionamiento, estructura y medios de la institución militar, y se oriente a la generación de una estructura de fuerzas y un catálogo de capacidades útiles para la guerra del siglo XXI. 